This item was submitted to Loughborough's Research Repository by the author.

Items in Figshare are protected by copyright, with all rights reserved, unless otherwise indicated.

\title{
Is a single or double arm technique more advantageous in triple jumping?
}

PLEASE CITE THE PUBLISHED VERSION

http://dx.doi.org/10.1016/j.jbiomech.2010.07.030

\section{PUBLISHER}

(c) Elsevier

VERSION

AM (Accepted Manuscript)

\section{LICENCE}

CC BY-NC-ND 4.0

\section{REPOSITORY RECORD}

Allen, Samuel J., Mark A. King, and Maurice R. Yeadon. 2019. "Is a Single or Double Arm Technique More Advantageous in Triple Jumping?". figshare. https://hdl.handle.net/2134/7633. 
This item was submitted to Loughborough's Institutional Repository (https://dspace.lboro.ac.uk/) by the author and is made available under the following Creative Commons Licence conditions.

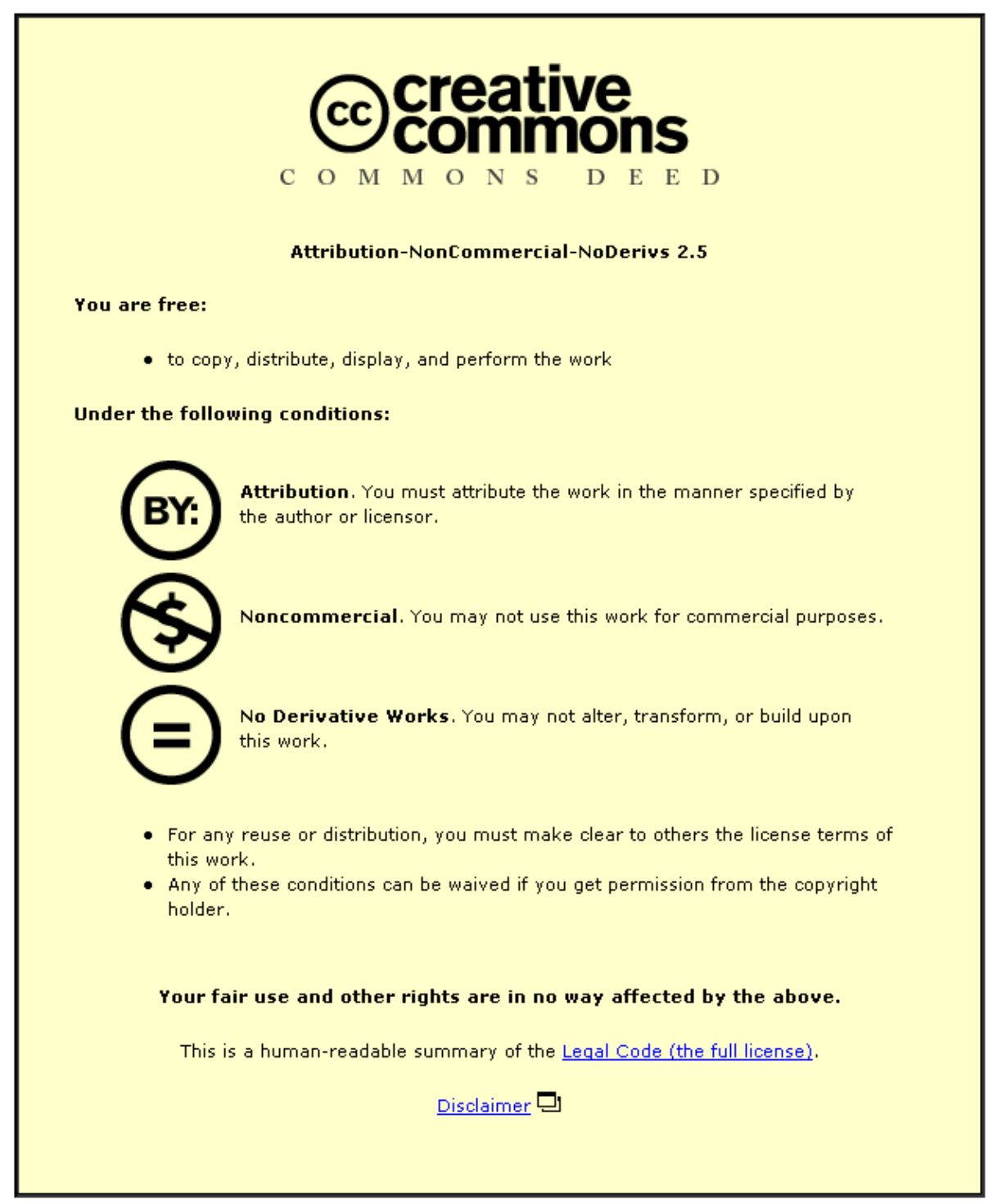

For the full text of this licence, please go to: http://creativecommons.org/licenses/by-nc-nd/2.5/ 


\title{
Is a single or double arm technique more advantageous in triple jumping?
}

\author{
S.J. Allen, M.A. King, and M.R. Yeadon
}

School of Sport, Exercise and Health Sciences, Loughborough University, Loughborough LE11 3TU, UK

\begin{abstract}
Triple jumpers employ either an asymmetrical 'single-arm' action or symmetrical 'double-arm' action in the takeoff of each phase of the jump. This study investigated which technique is more beneficial in each phase using computer simulation. Kinematic data were obtained from an entire triple jump using a Vicon automatic motion capture system. A planar 13-segment torque-driven subject-specific computer simulation model was evaluated by varying torque generator activation timings using a genetic algorithm in order to match performance data. The matching produced a close agreement between simulation and performance, with differences of 3.8\%, 2.7\%, and 3.1\% for the hop, step, and jump phases respectively. Each phase was optimised for jump distance and an increase in jump distance beyond the matched simulations of $3.3 \%, 11.1 \%$, and $8.2 \%$ was obtained for the hop, step, and jump respectively. The optimised technique used symmetrical shoulder flexion whereas the triple jumper had used an asymmetrical arm technique. This arm action put the leg extensors into slower concentric conditions allowing greater extensor torques to be produced. The main increases in work came at the joints of the stance leg but the largest increases in angular impulse came at the shoulder joints, indicating the importance of both measures when assessing the impact of individual joint actions on changes in technique. Possible benefits of the double-arm technique include: cushioning the stance leg during impact; raising the centre of mass of the body at takeoff; facilitating an increase in kinetic energy at takeoff; allowing a re-orientation of the body during flight.
\end{abstract}

Keywords: computer simulation, torque, triple jump, arm, technique

\section{INTRODUCTION}

When Jonathan Edwards improved his best performance by $0.85 \mathrm{~m}$ in breaking the triple jump world record three times in 1995, he attributed his improvement to the adoption of a symmetrical 'double-arm' technique (Edwards, 2009). Hay (1992) stated that, 'The arm action used to takeoff into each of the three phases has been the source of considerable debate over the last 2-3 decades'. Arm techniques employed during the takeoff phases of the triple jump can be broadly split into two types: the single-arm technique in which the arms move in an asymmetrical fashion as they do in running; and the double-arm technique, which involves a symmetrical flexion of the upper arms during takeoff, starting from an extended position. Athletes can employ any combination of these two techniques over the three phases of the triple jump. There has, however, been very little consideration of arm technique in the literature, with analysis limited to observation of the techniques employed by elite performers (Jarver and Boase, 1984; Masters, 1986) and no attempt to assess quantitatively which technique is best and why.

It has been shown that jumping performance is improved with an arm swing in vertical jumps (Harman et al., 1990; Lees et al., 2004), and in horizontal jumps (Ashby and Heergard, 2002; Hara et al., 2008). Two main mechanisms were proposed by Harman et al. (1990) for this performance improvement: that the energy generated by the shoulder musculature directly contributes to the energy of the jump; and that the accelerations of the arms cause the leg musculature to be forced into slower concentric conditions, allowing the production of greater torque.

The aim of this study was to develop a realistic computer simulation model of triple jumping in order to investigate which arm technique is associated with optimal performance. 


\section{METHODS}

Kinematic and force data were gathered at the Loughborough University indoor High Performance Athletics Centre (HiPAC) from a male triple jumper of national standard (age: 22 years; mass: $72.6 \mathrm{~kg}$; height: $1.82 \mathrm{~m}$; best performance: $14.35 \mathrm{~m}$ ). The study was carried out in accordance with the Loughborough University Ethical Advisory Committee guidelines. Forty-five $25 \mathrm{~mm}$ retroreflective markers were placed in positions on the jumper's body in order that locations of joint centres could be calculated. Eighteen Vicon MX cameras covered a volume of $18 \mathrm{~m} \times 2 \mathrm{~m}$ x $2.5 \mathrm{~m}$ spanning the last stride of the approach and the full triple jump. Data were captured at 240 $\mathrm{Hz}$ during a single triple jump performance of $13.00 \mathrm{~m}$. Orientation, defined as the angle of the trunk in the global reference frame, and configuration angles were calculated by considering the joint centre coordinates in the sagittal plane. Quintic splines (Wood and Jennings, 1979) were fitted to the time histories of these angles for input to the simulation model.

A 13-segment planar torque-driven computer simulation model of triple jumping was developed to investigate triple jumping technique (Figure 1). The 13 segments represented: head + trunk, two upper arms, two forearms and hands, two thighs, two shanks, two 2-segment feet, with wobbling masses within the shanks, thighs, and torso. Each foot had three points of contact with the ground at the heel, ball (metatarsophalangeal joint), and toe. The model was driven by torque generators consisting of contractile components and series elastic components which were employed to flex and extend the shoulder, hip, knee, ankle, and ball joints. The elbow joints were angle-driven since it was assumed they would behave similarly to recorded performances.

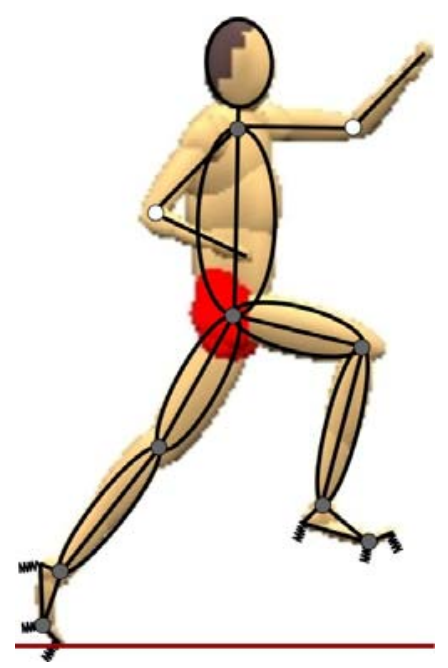

Figure 1. Thirteen-segment simulation model with wobbling masses within the shank, thigh, and trunk segments, torque drivers at the ball, ankle, knee, hip, and shoulder joints (grey circles), angle drivers at the elbow joints (white circles), and spring-dampers at three points on each foot.

Subject-specific torque and inertia parameters were calculated from measurements taken from an elite triple jumper. Maximal voluntary joint torque data were obtained assuming bilateral symmetry using an Isocom isovelocity dynamometer for flexion and extension of the ankle, knee, hip, and shoulder on the right side of the body (King et al., 2006). Due to the difficulties in measuring joint torques at the ball joint, profiles from the ankle joint were used, with maximum isometric torques reduced by a factor of three. In addition to active torque generators, the ankle, knee, and hip had passive elements (Riener and Edrich, 1999) which produced restorative torques at the extremes of range, acting to stop the limb exceeding anatomical limits. Ninety-five anthropometric measurements were taken and used as input to the inertia model of Yeadon (1990) in order to calculate subject-specific segmental inertia parameters.

Non-linear spring-dampers connected the ends of the wobbling and rigid elements (Pain and Challis, 2001): 


$$
\mathbf{R}_{\mathrm{i}}=\left(-\mathrm{k}_{\mathrm{i}} \cdot\left|\mathbf{r}_{\mathrm{i}}\right|^{3}-\mathrm{d}_{\mathrm{i}} \cdot\left|\dot{\mathbf{r}}_{\mathrm{i}}\right|\right) \cdot \hat{\mathbf{r}}_{\mathrm{i}}, \quad(\text { for } \mathrm{i}=1,3)
$$

where $\mathbf{R}$ is a force vector, $\mathbf{r}$ is a vector defining the position of the point of attachment on the wobbling mass from that on the rigid segment, $\hat{\mathbf{r}}$ is a unit vector in the direction of $\mathbf{r},|\mathbf{r}|$ is the magnitude of $\mathbf{r},|\dot{\mathbf{r}}|$ is the derivative of $|\mathbf{r}|, \mathrm{k}$ and $\mathrm{d}$ are stiffness and damping coefficients respectively, and i represents the location of the wobbling mass.

The horizontal and vertical forces at the foot were both modelled as modified linear springdampers situated at the heel, ball, and toe of each foot:

$$
\begin{array}{ll}
\mathrm{R}_{\mathrm{yi}}=-\mathrm{k}_{\mathrm{yi}} \cdot \mathrm{y}_{\mathrm{i}}-\mathrm{d}_{\mathrm{yi}} \cdot \dot{\mathrm{y}}_{\mathrm{i}} \cdot\left|\mathrm{y}_{\mathrm{i}}\right|, \quad & (\text { for } \mathrm{i}=1,3) \\
\mathrm{R}_{\mathrm{xi}}=\left(-\mathrm{k}_{\mathrm{xi}} \cdot \mathrm{x}_{\mathrm{i}}-\mathrm{d}_{\mathrm{xi}} \cdot \dot{\mathrm{x}}_{\mathrm{i}}\right) \cdot \mathrm{R}_{\mathrm{yi}}, & (\text { for } \mathrm{i}=1,3)
\end{array}
$$

where $\mathrm{R}_{\mathrm{x}}$ and $\mathrm{R}_{\mathrm{y}}$ are the horizontal and vertical forces, $x$ is the horizontal displacement from the initial ground contact point, $y$ is the vertical displacement with respect to the floor, $\dot{x}$ and $\dot{y}$ are the derivatives of $\mathrm{x}$ and $\mathrm{y}, \mathrm{k}_{\mathrm{x}}$ and $\mathrm{k}_{\mathrm{y}}, \mathrm{d}_{\mathrm{x}}$ and $\mathrm{d}_{\mathrm{y}}$ are the stiffness and damping coefficients respectively, and $\mathrm{i}$ represents the point of contact on the foot.

Optimisation was used in three different ways: angle-driven simulations were matched to performance data in order to determine viscoelastic parameter values; torque-driven simulations were matched to performance data in order to assess the accuracy of the model; technique was optimised using torque-driven simulations in order to maximise phase distances. In each of these optimisations each phase of the triple jump was treated independently and had no bearing on the subsequent phases. In the angle-driven simulations the stance and airborne phases were simulated for each phase. In the torque-driven simulations the stance phase was simulated while in the airborne phase it was assumed that the model would undergo the same configuration changes as in the performance. The associated orientation changes were determined by running an angle-driven simulation with no angular momentum and this allowed the landing orientation in each phase to be calculated using the orientation, angular and linear momenta at takeoff as determined in the torquedriven simulation.

A common set of viscoelastic parameters representing the attachments of the wobbling masses and the foot-ground interface was determined for the three phases of the triple jump using an adaptation of the method of Wilson et al. (2006). The simulation model was angle-driven using performance data and 27 parameters were varied using a simulated annealing algorithm (Corana et al., 1987) in order to minimise the difference between simulation and performance. These 27 parameters comprised: 12 stiffness and damping coefficients at the foot; six stiffness and damping coefficients at the wobbling masses; and nine kinematic parameters representing initial angular and linear velocities of the whole body in each phase. The objective difference function was the root mean square (RMS) of four parts: $\mathrm{S}_{1}$ - \% difference in horizontal velocity of COM at takeoff; $\mathrm{S}_{2}$ $\%$ difference in vertical velocity of COM at takeoff; $S_{3}$ - overall RMS difference in (trunk) orientation in degrees in the contact and airborne phases; $\mathrm{S}_{4}-\%$ absolute difference in time of contact. In all cases $1^{\circ}$ was considered to be equivalent to $1 \%$ and objective difference function values were reported as \%.

The torque-driven model was evaluated by assessing how accurately a simulation could match performance data for each phase individually. This simulation was found by varying 77 torque generator activation timings and seven initial kinematic conditions in order to minimise a difference function between simulation and performance data using a genetic algorithm (Goldberg, 1989). The seven initial kinematic conditions comprised the orientation angle; configuration angles at the ankle, knee, and hip; and the angular and linear velocities of the whole body. At any given time during a simulation the activation level of each torque generator was governed by a quintic function with zero accelerations and velocities at the endpoints (Yeadon and Hiley, 2000). The minimum 
time in which activation could ramp up from zero to maximum was 70 ms (Yeadon et al., 2010). Torque profiles were either: ramp up (four parameters); ramp up and ramp down (up to seven parameters), or ramp down and ramp up (seven parameters), and were consistent across the three phases. These parameters defined: the time of onset of the activation ramp; the initial level of activation; the time taken to ramp up from zero to maximum; the maximum level of activation achieved; the time of onset of the second ramp; the time taken to ramp down from maximum to zero; and the final level of activation. The 77 torque generator activation timings comprised parameters from the profiles of all the torque-driven joints. The type of profile of each torque generator was estimated using joint torques calculated using inverse dynamics from the angledriven model, and muscle activations reported in the literature for triple jumping (Perttunen et al., 2000). The ankle and ball joints in the free leg had constant activation profiles since they were expected to have a small influence on the performance of the model and were not included in the matching process. The ball joint of the stance leg was assumed to have only extensor activation. Levels of activation at touchdown were limited to 0.5 times maximum in all joints except for the free hip flexor where touchdown activation was allowed to take any value up to maximum since the flexion movement could be initiated prior to impact.

The objective function for each matched torque-driven simulation was the RMS of six parts: $S_{1}$ - \% difference in horizontal velocity of COM at takeoff; $S_{2}$ - \% difference in vertical velocity of $\mathrm{COM}$ at takeoff; $\mathrm{S}_{3}$ - overall RMS difference in (trunk) orientation in degrees during ground contact; $\mathrm{S}_{4}$ - overall RMS difference in whole-body configuration in degrees during ground contact; $S_{5}-\%$ absolute difference in time of contact; $S_{6}$ - absolute difference in orientation at touchdown of the subsequent phase in degrees. The latter component was calculated using the simulation angular momentum at takeoff, and the orientation change associated with the performance configuration time history during the airborne phase.

Technique was optimised in order to maximise distance in each phase of the triple jump. The seven kinematic initial conditions were the same as for the matched simulation and the 77 activation parameters were varied by the genetic algorithm. Penalties were imposed if the joints exceeded anatomical limits, or if the calculated landing orientation was more than $5^{\circ}$ from the measured landing orientation. Each phase distance $\left(\mathrm{d}_{\text {phase }}\right)$ comprised three components (Figure 2): The takeoff distance $\left(\mathrm{d}_{\text {takeoff }}\right)$; the flight distance $\left(\mathrm{d}_{\text {flight }}\right)$; and the landing distance $\left(\mathrm{d}_{\text {landing }}\right)$.

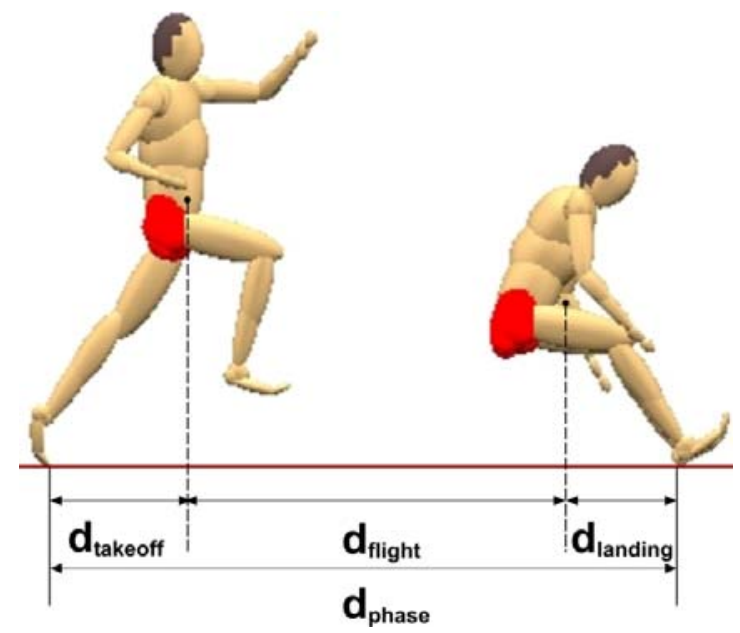

Figure 2. The three components of a phase distance $\left(\mathrm{d}_{\text {phase }}\right)$ : the takeoff distance $\left(\mathrm{d}_{\text {takeoff }}\right)$ is the horizontal distance from the toe of the stance leg to the COM at takeoff; the flight distance $\left(\mathrm{d}_{\text {flight }}\right)$ is the horizontal distance travelled by the COM whilst the athlete is airborne; and the landing distance $\left(\mathrm{d}_{\text {landing }}\right)$ is the horizontal distance from the COM to the toe of the stance leg at touchdown, or in the case of the jump, the most posterior of the two heels at landing. 


\section{RESULTS}

Simulation results from the angle-driven model indicated that the optimised viscoelastic parameters (Table 1) led to accurate model kinematics and were therefore acceptable for use in further simulations of the triple jump. Each phase was well matched, with difference function values of $2.2 \%, 1.0 \%$ and $2.4 \%$ for the hop, step, and jump phases respectively.

\begin{tabular}{lr}
$\begin{array}{l}\text { Table } 1 . \\
\text { Viscoelastic parameters obtained from the angle-driven } \\
\text { simulations }\end{array}$ \\
\hline parameter & value \\
\hline vertical toe stiffness $-\mathrm{k}_{\mathrm{y} 1}\left(\mathrm{Nm}^{-1}\right)$ & 453260 \\
vertical ball stiffness $-\mathrm{k}_{\mathrm{y} 2}\left(\mathrm{Nm}^{-1}\right)$ & 147360 \\
vertical heel stiffness $-\mathrm{k}_{\mathrm{y} 3}\left(\mathrm{Nm}^{-1}\right)$ & 291880 \\
vertical toe damping $-\mathrm{d}_{\mathrm{y} 1}\left(\mathrm{Nsm}^{-2}\right)$ & 463390 \\
vertical ball damping $-\mathrm{d}_{\mathrm{y} 2}\left(\mathrm{Nsm}^{-2}\right)$ & 113740 \\
vertical heel damping $-\mathrm{d}_{\mathrm{y} 3}\left(\mathrm{Nsm}^{-2}\right)$ & 249560 \\
\hline horizontal toe stiffness $-\mathrm{k}_{\mathrm{x} 1}\left(\mathrm{~m}^{-1}\right)$ & 126 \\
horizontal ball stiffness $-\mathrm{k}_{\mathrm{x} 2}\left(\mathrm{~m}^{-1}\right)$ & 107 \\
horizontal heel stiffness $-\mathrm{k}_{\mathrm{x} 3}\left(\mathrm{~m}^{-1}\right)$ & 0.10 \\
horizontal toe damping $-\mathrm{d}_{\mathrm{x} 1}\left(\mathrm{sm}^{-1}\right)$ & 1.15 \\
horizontal ball damping $-\mathrm{d}_{\mathrm{x} 2}\left(\mathrm{sm}^{-1}\right)$ & 1.40 \\
horizontal heel damping $-\mathrm{d}_{\mathrm{x} 3}\left(\mathrm{sm}^{-1}\right)$ & 196 \\
\hline wobbling mass stiffness shank $-\mathrm{k}_{1}\left(\mathrm{Nm}^{-3}\right)$ & 636850000 \\
wobbling mass stiffness thigh $-\mathrm{k}_{2}\left(\mathrm{Nm}^{-3}\right)$ & 11392000 \\
wobbling mass stiffness torso $-\mathrm{k}_{3}\left(\mathrm{Nm}^{-3}\right)$ & 1885100 \\
wobbling mass damping shank $-\mathrm{d}_{1}\left(\mathrm{Nsm}^{-1}\right)$ & 1734 \\
wobbling mass damping thigh $-\mathrm{d}_{2}\left(\mathrm{Nsm}^{-1}\right)$ & 90 \\
wobbling mass damping torso $-\mathrm{d}_{3}\left(\mathrm{Nsm}^{-1}\right)$ & \\
\hline
\end{tabular}

The evaluation of the torque-driven model led to good correspondence with performance data (Figure 3), demonstrating sufficient complexity for subsequent optimisation of performance. Difference function values were $3.8 \%, 2.7 \%$ and $3.1 \%$ for the hop, step, and jump phases respectively. The passive torques which came into play at the extremes of the anatomical ranges were relatively small $(<20 \mathrm{Nm})$. 
b

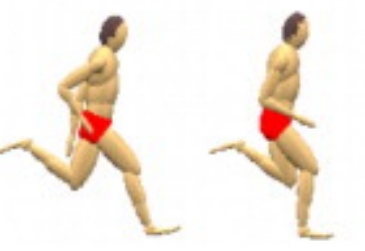

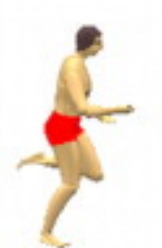

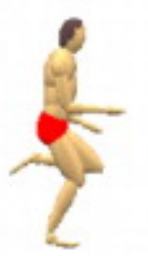

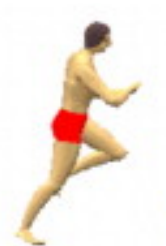
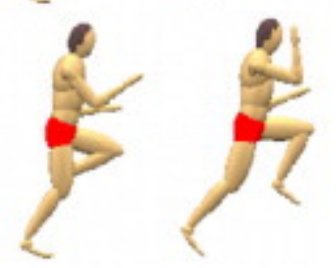

2 a
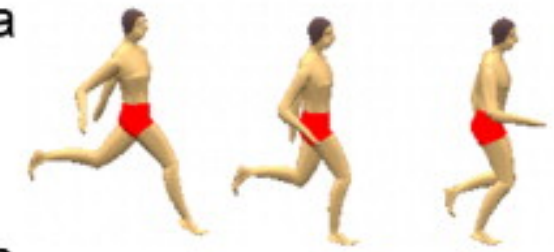

$b$
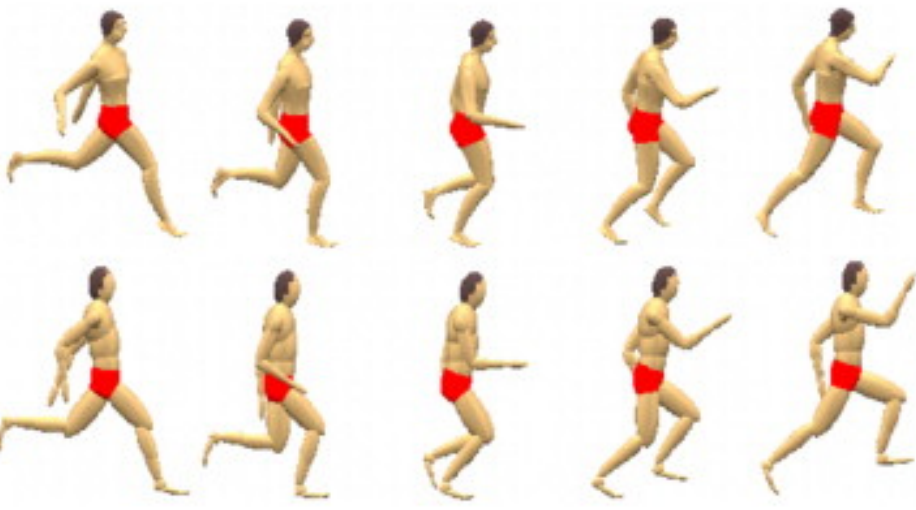

3
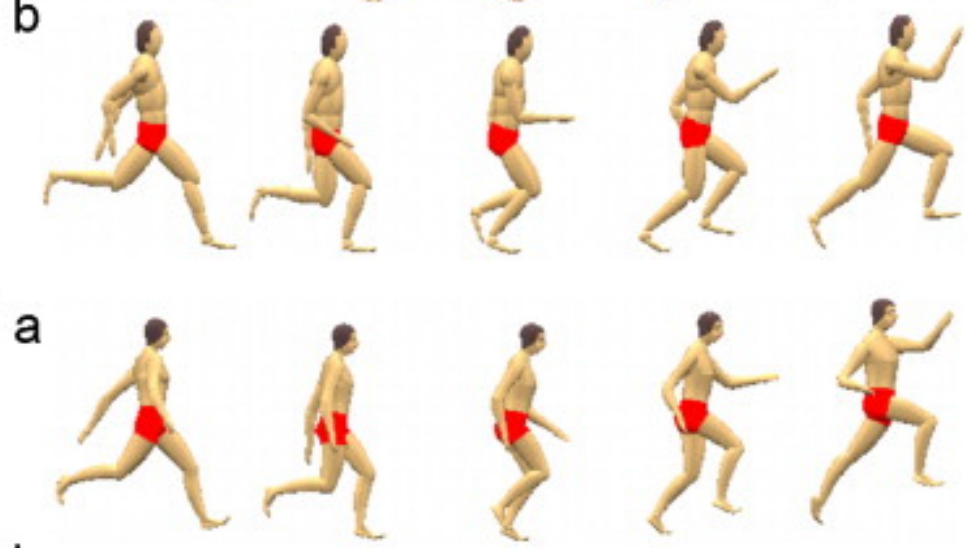

b
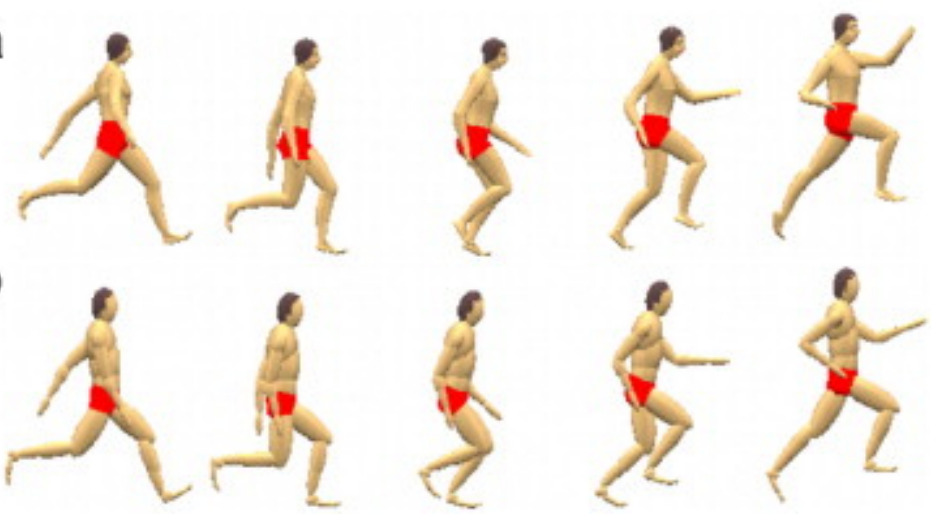

Figure 3. Performance (a) and matched (b) simulations of the hop (1), step (2), and jump (3) phases.

Optimisation of technique in each phase yielded an increase in jump distance from the matched simulations of 3.3\%, 11.1\%, and $8.2 \%$ for the hop, step, and jump respectively (Table 2). The landing distance was fixed for all simulations so improvements could only come from $\mathrm{d}_{\text {takeoff }}$ and $\mathrm{d}_{\text {flight. }}$ In each phase the optimisation process chose a symmetrical shoulder flexion, whereas the jumper employed an asymmetrical technique (Figure 4). 
Table 2. Differences in each component of phase distance between matched and optimised simulations of each phase

\begin{tabular}{cccccc}
\hline \multirow{2}{*}{ phase } & simulation & $\begin{array}{c}\mathrm{d}_{\text {takeoff }} \\
(\mathrm{m})\end{array}$ & $\begin{array}{c}\mathrm{d}_{\text {flight }} \\
(\mathrm{m})\end{array}$ & $\begin{array}{c}\mathrm{d}_{\text {landing }} \\
(\mathrm{m})\end{array}$ & $\begin{array}{c}\mathrm{d}_{\text {phase }} \\
(\mathrm{m})\end{array}$ \\
\hline \multirow{2}{*}{ hop } & matched & 0.43 & 3.21 & 0.65 & 4.29 \\
& optimised & 0.55 & 3.23 & 0.65 & 4.43 \\
& difference & 0.12 & 0.02 & 0.00 & 0.14 \\
\hline \multirow{2}{*}{ step } & matched & 0.58 & 2.59 & 0.72 & 3.89 \\
& optimised & 0.62 & 2.98 & 0.72 & 4.32 \\
& difference & 0.04 & 0.39 & 0.00 & 0.43 \\
\hline \multirow{2}{*}{ jump } & matched & 0.47 & 3.37 & 0.44 & 4.28 \\
& optimised & 0.55 & 3.64 & 0.44 & 4.63 \\
& difference & 0.08 & 0.27 & 0.00 & 0.35 \\
\hline
\end{tabular}

The optimised techniques resulted in longer ground contact time $(0.018 \mathrm{~s}, 0.008 \mathrm{~s}$, and 0.009 s) (Table 3), increased height of the COM at takeoff $(0.04 \mathrm{~m}, 0.04 \mathrm{~m}$, and $0.02 \mathrm{~m})$ (Table 3), and increased $\mathrm{d}_{\text {takeoff }}$ for each phase (Table 2). Optimised techniques also showed increased vertical reaction impulses $(2.9 \%, 4.8 \%$, and $3.3 \%)$ leading to increases in vertical velocity and projection angle of the COM at takeoff in the step and jump phases $\left(0.22 \mathrm{~m} / \mathrm{s}\right.$ and $1.9^{\circ}, 0.21 \mathrm{~m} / \mathrm{s}$ and $\left.0.3^{\circ}\right)$ but not the hop phase $\left(-0.05 \mathrm{~m} / \mathrm{s}\right.$ and $\left.-0.7^{\circ}\right)$ due to the larger increase in hop contact time.

Table 3. Ground contact times and COM heights at takeoff in matched and optimised simulations of each phase

\begin{tabular}{clcc}
\hline phase & simulation & contact time $(\mathrm{s})$ & COM height $(\mathrm{m})$ \\
\hline \multirow{4}{*}{ hop } & matched & 142 & 1.06 \\
& optimised & 160 & 1.10 \\
& difference & 18 & 0.04 \\
\hline \multirow{4}{*}{ step } & matched & 183 & 1.06 \\
& optimised & 191 & 1.10 \\
& difference & 8 & 0.04 \\
\hline \multirow{4}{*}{ jump } & matched & 203 & 1.07 \\
& optimised & 212 & 1.09 \\
& difference & 9 & 0.02 \\
\hline
\end{tabular}


1

a

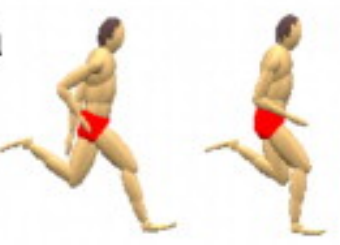

b
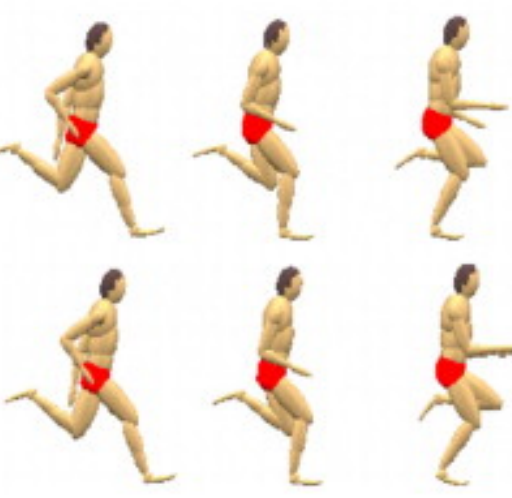

2

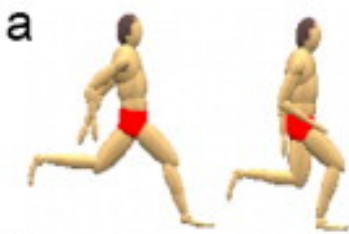

b

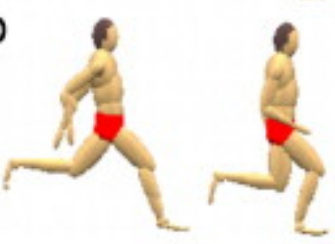

3 a

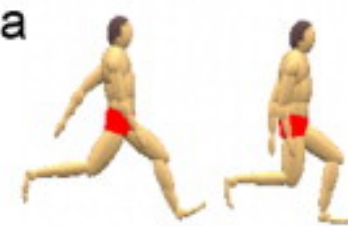

b

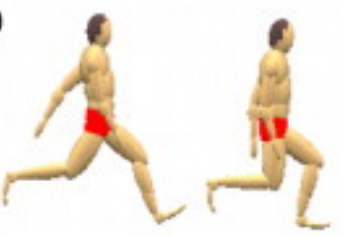

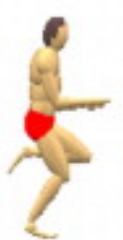
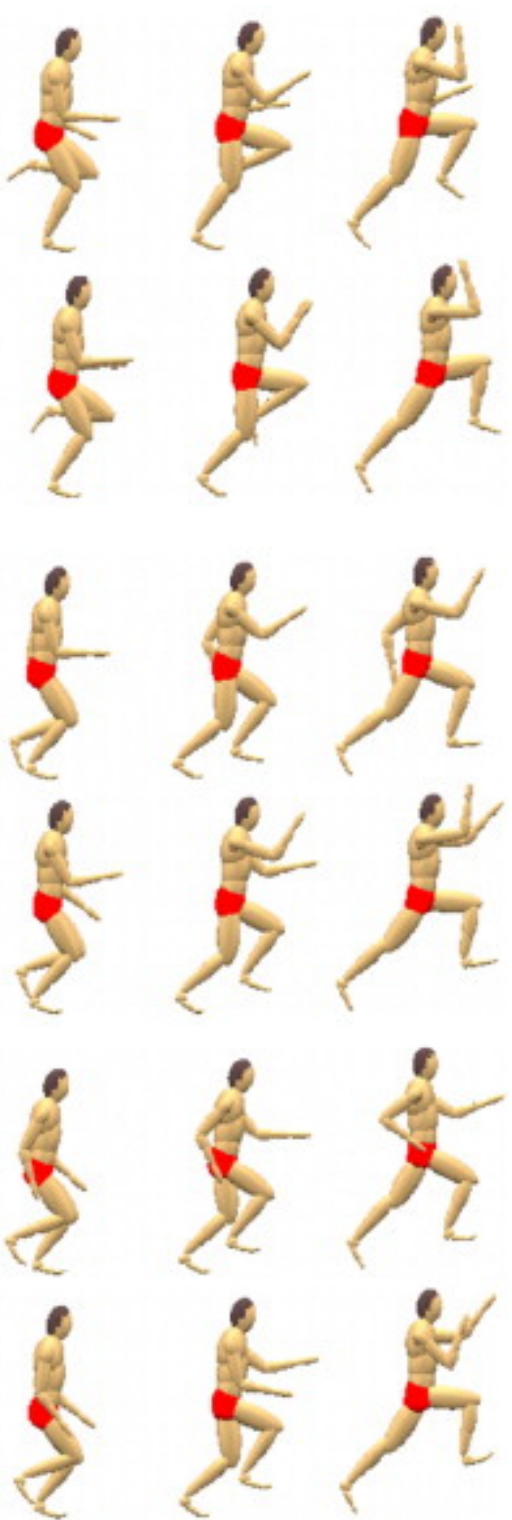

Figure 4. Matched (a) and optimised (b) simulations of the hop (1), step (2), and jump (3) phases.

\section{DISCUSSION}

The most marked kinematic differences between the matched and optimised simulations can be seen at the shoulder joints. The angle of the shoulder joint contralateral to the stance hip in the optimised simulations deviated considerably from that of the matched simulations in each phase. The optimised simulations used a symmetrical flexion of both shoulder joints, whereas the matched simulation showed an asymmetrical arm movement (Figure 3). In addition to this symmetrical arm action there was also a general trend towards greater extension of the joints of the stance leg and a greater flexion of the free hip in the optimised simulations. This all acted to increase ground contact time, facilitated the increase in vertical impulse that was seen in each phase, and also acted to increase $d_{\text {takeoff }}$ and raise the whole body COM at takeoff in each phase (Ashby and Delp, 2006). There was an overall increase in work done of $70 \mathrm{~J}, 103 \mathrm{~J}$, and $130 \mathrm{~J}$ in the hop, step, and jump phases by the torque-driven joints from the matched to the optimised simulations. The largest increases in work were at the stance hip in the step and jump phases, and this was also a large contributor to the increase in work in the hop phase. This finding is in agreement with that of Cheng et al. (2008) who showed that an arm swing facilitated increases in work at the hip joint. 
Before the symmetrical arm technique is discussed further, it must be considered that asymmetrical arm actions might be employed by triple jumpers in order to counteract rotations about the longitudinal axis of the jumper. The planar nature of the model used in this study would not allow these rotations to be simulated. However the fact that some elite triple jumpers use a symmetrical double-arm technique (Hay, 1992) indicates that it is possible to control such rotations without utilising an asymmetrical technique and so the symmetrical technique is as viable as an asymmetrical technique in this respect.
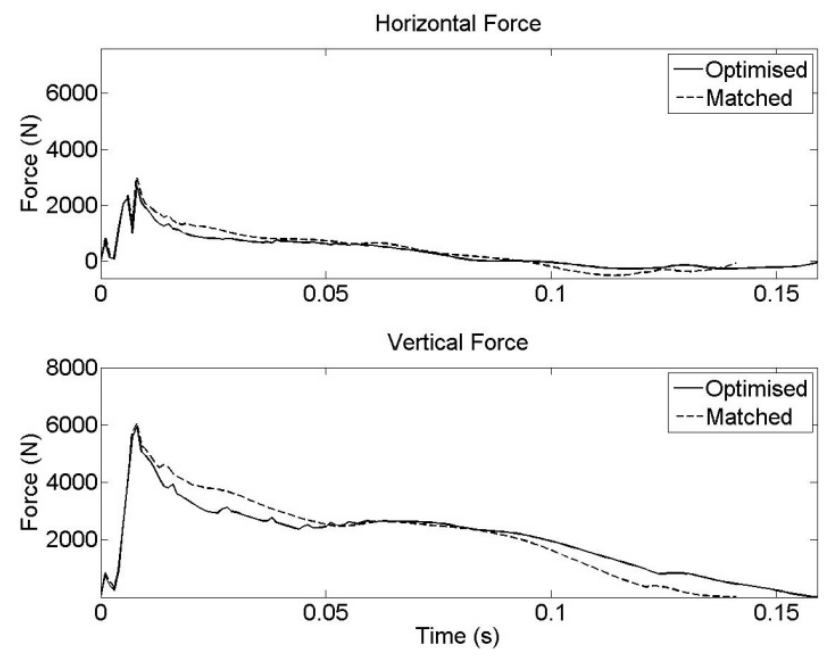

Figure 5. GRFs from matched (dashed lines) and optimised (solid lines) simulations of the hop phase.

The increased flexion at the shoulder joints in the optimised simulations manifested itself in increases in both $d_{\text {takeoff }}$ and the COM height at takeoff. All the optimised simulations showed an increased vertical impulse and ground contact time. An example of the origin of these increases in impulse can be seen in the force trace for the matched and optimised simulations of the hop phase (Figure 5) where the optimised simulation generated increased vertical ground reaction forces (GRFs) towards the end of the ground contact phase.
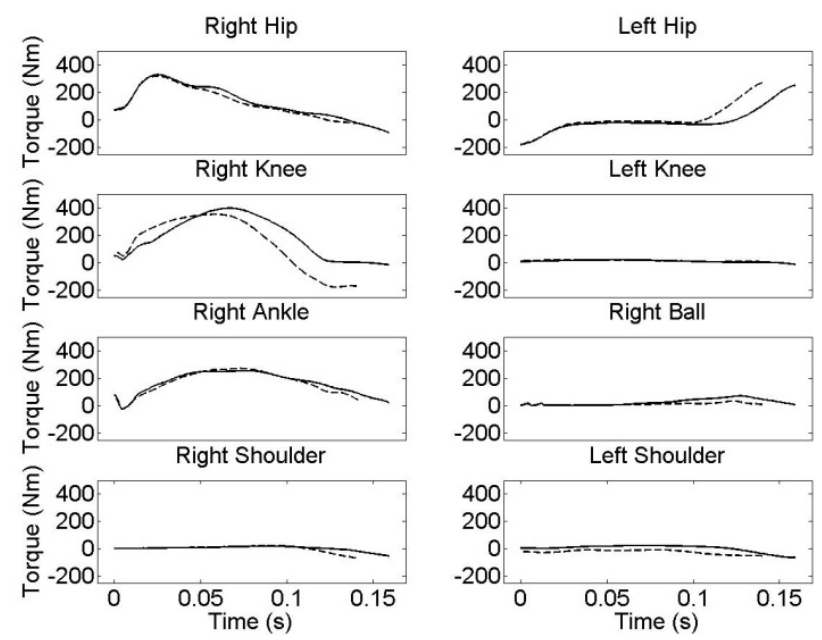

Figure 6. Joint torque time histories from matched (dashed lines) and optimised (solid lines) simulations of the hop phase (extension is positive except at shoulder joints).

With the exception of the shoulder joints there were no consistent trends in the changes in activation profiles from the matched to optimised simulations. The increases in joint torques seemed to come about largely because the torque generators were put in kinematic conditions that were more advantageous for torque production. The contributions of the angular velocities of the hip, knee, and ankle joints of the stance leg to torque production at these joints were compared for 
the time periods where extensor torque generators were maximally active, flexor torque generators inactive, and both simulations were in contact with the ground. Since the contribution of angular velocity to torque production was multiplicative (Yeadon et al., 2006) the differences in this contribution were calculated as average percentages of the maximum isometric contribution. In most cases the optimised simulations showed a larger contribution of angular velocity to torque production at the hip, knee and ankle joints than in the matched simulations in the hop (5.5\%, $0.0 \%$, $7.8 \%)$, step $(0.6 \%, 2.2 \%, 5.0 \%)$, and jump $(-1.3 \%, 8.3 \%, 5.1 \%)$ phases. These results provide evidence for the theory that improvements in performance due to arm swing come about due to the muscles of the support leg being put into slower concentric conditions.
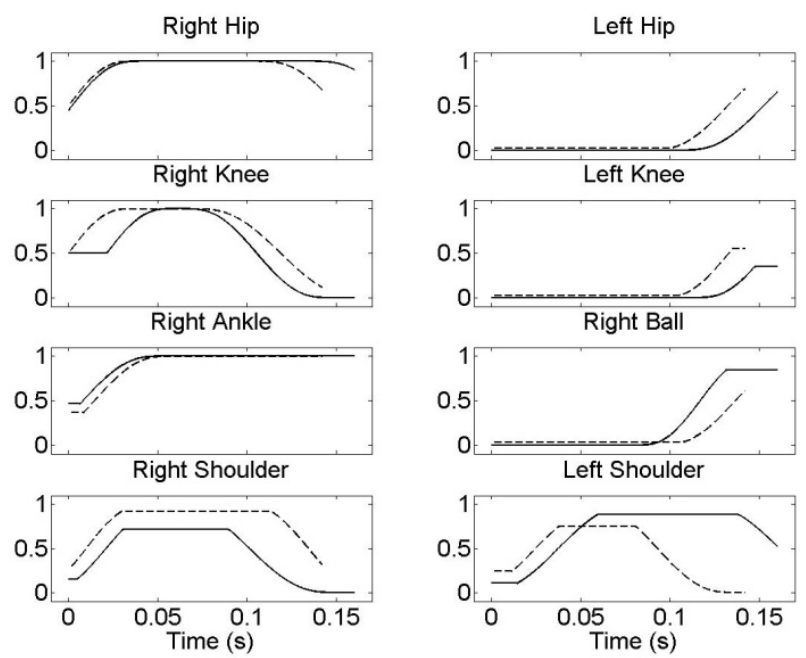

Figure 7. Activation time histories for joint extensors* from matched (dashed lines) and optimised (solid lines) simulations of the hop phase (*shoulders show flexors).
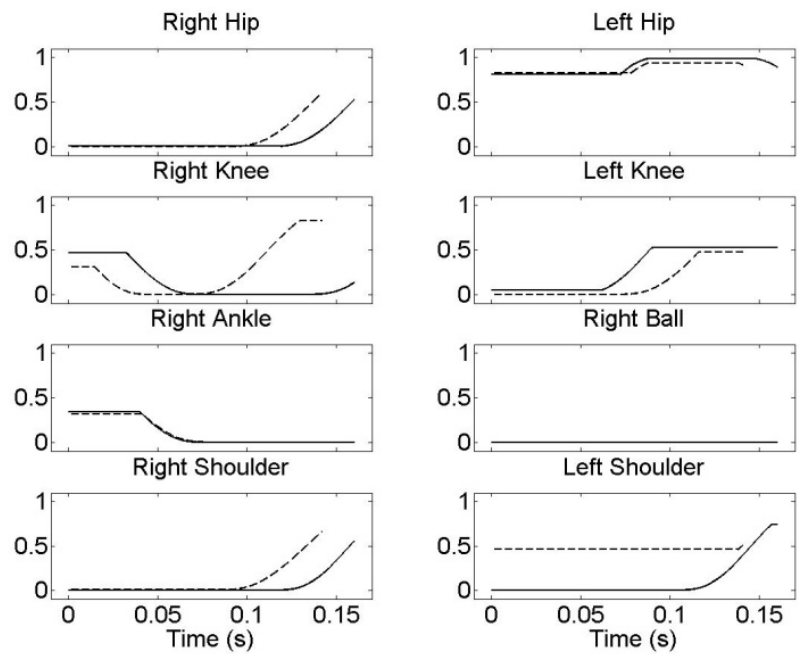

Figure 8. Activation time histories for joint flexors* from matched (dashed lines) and optimised (solid lines) simulations of the hop phase (*shoulders show extensors).

In essence the two main mechanisms that have been proposed to explain how an arm swing improves jump performance (Harman et al., 1990) are biologically similar: any accelerations generated by the shoulder joints must be transmitted to the floor in order to increase the GRFs, if the velocity of the COM is to be increased. In order for this to happen the muscles of the legs must generate greater joint torques to prevent the joints from collapsing. Assuming that the muscles are maximally activated, this increase in torque must therefore come about due to the muscles of the leg being put in either faster eccentric, or slower concentric, conditions. However, only the latter will 
result in an increase in mechanical work. Slower concentric conditions will also lead to an increased contact time which may facilitate an increase in linear impulse.

Another likely benefit of the arm swing in triple jumping is the 'cushioning' of the stance leg on impact. It has been shown that arm movements act to cushion the impact on the stance leg in a high jump takeoff (Dapena and Chung, 1988) in which the athlete employed a double-arm technique similar to that seen in triple jumping. This cushioning occurs in the opposite fashion to the mechanism described above during the period when the shoulders are flexing from a hyperextended position shortly after touchdown. While this will put the stance leg extensors into slower eccentric conditions this will not reduce the extensor torques greatly due to the torque plateau in eccentric mode.

The main increase in work came at the stance knee joint in the hop phase (31 J) and the stance hip joints in the step and jump phases (64 J and $59 \mathrm{~J}$ ). This is again consistent with the idea that the arm action puts the stance leg into slower concentric conditions. Increases in work were often associated with increased angular impulses. In each phase the double-arm technique in the optimised simulations allowed the shoulder joints to do more work. These increases in work were typically modest ( $5 \mathrm{~J}, 21 \mathrm{~J}$, and $9 \mathrm{~J}$ ), although the increases in angular impulse were large (3.9 Nms, 8.2 Nms, and 5.6 Nms) and in the case of the step and jump phases, the largest of any joint. This indicates the importance of considering both measures when assessing technical changes in performance. Work is a scalar quantity and hence it is difficult to say whether an increase will benefit performance. Even if the arms are doing additional work, the manifestation of this work in the GRFs, and therefore the acceleration of the COM, is dependent on the force / velocity relationship of the lower limb musculature and its ability to withstand any forces imposed by the accelerations of the arms. The choice of measure is especially important when analysing jumps with initial horizontal velocity, since the eccentric actions of the leg musculature can contribute directly to changes in the vertical velocity of the COM (Dapena and Chung, 1988).

In the hop and step phases the performance of the jumper might have been limited due to the requirement that the vertical velocity at landing into the subsequent takeoff should not be too large. Since each phase was optimised individually, with initial conditions taken from the measured performance, this could not be taken into consideration. However, the fact that the jump takeoff, where there was no such requirement, showed a substantial improvement in performance when optimised might indicate that this was not the case.

Using angle-driven elbow joints might be considered to be a limitation since these joints may behave differently in single arm and double arm actions. Indeed if the elbow joints were to be torque-driven then equal elbow angles at takeoff might be expected in the optimised simulations shown in Figure 4. Such optimisations might produce marginally better performance than the current optimisations but are likely to be very similar in the selected arm action.

Bilateral symmetry was assumed in the determination of joint torque parameters. It might be argued that triple jumping does not necessarily encourage or require symmetrical leg strength. On the other hand each leg is used for stance in one phase or other and training typically incorporates a large number of bilateral exercises which will ensure that strength does not differ substantially between legs.

Since the optimisations were started from the matched simulations which employed asymmetrical arm actions, it might be expected that a local optimum technique employing asymmetrical arms would be found. The fact that the optimised technique changed this to a symmetrical arm movement in each phase is striking and suggests that a global optimum was found. The optimisation of the complete triple jump with the result of one phase leading into the next remains a challenging but feasible prospect. We hypothesise that the extra freedom in initial conditions allowed by this will facilitate further improvements in simulated performance. 


\section{REFERENCES}

Ashby, B.M., Delp, S.L., 2006. Optimal control simulations reveal mechanisms by which arm movement improves standing long jump performance. Journal of Biomechanics 39, 17261734.

Ashby, B.M., Heergard, J.H. 2002., Role of arm motion in the standing long jump. Journal of Biomechanics 35, 1631-1637.

Cheng, K., Wang, C., Chen, H., Wu, C., Chiu, H., 2008. The mechanisms that enable arm motion to enhance vertical jump performance - A simulation study. Journal of Biomechanics 41, 18471854.

Corana, A., Marchesi, M., Martini, C., Ridella, S., 1987. Minimising multimodal functions of continuous variables with the "Simulated Annealing" algorithm. ACM Transactions on Mathematical Software 12, 262-280.

Dapena, J., Chung, C.S., 1988. Vertical and radial motions of the body during the take-off phase of high jumping. Medicine and Science in Sports and Exercise 20, 290-302.

Edwards, J., 2009. http://www.iaaf.org/GLE09/news/newsid=51022.html

Goldberg, D.E., (1989). Genetic algorithms in search, optimization and machine learning. First edn. Boston, MA: Addison-Wesley.

Hara, M., Shibayama, A., Arakawa, H., Fukashiro, S., 2008. Effect of arm swing direction on forward and backward jump performance. Journal of Biomechanics 41, 2806-2815.

Harman, E.A., Rosenstein, M.T., Frykman, P.N., Rosenstein, R.M., 1990. The effect of arms and countermovement on vertical jumping. Medicine and Science in Sports and Exercise 22, 825833.

Hay, J.G., 1992. The biomechanics of the triple jump: a review. Journal of Sports Sciences 10, 343378.

Jarver, J., Boase, G., 1984. Triple jump. Modern Athlete and Coach 22, 6-7.

King, M.A., Wilson, C., Yeadon, M.R., 2006. Evaluation of a torque-driven model of jumping for height. Journal of Applied Biomechanics 22, 264-274.

Lees, A., Vanrenterghem, J., Clercq, D.D., 2004. Understanding how an arm swing enhances performance in the vertical jump. Journal of Biomechanics 37, 1929-1940.

Masters, A. 1986. A coach’s view - arms in the triple jump takeoff. Modern Athlete and Coach 24, 33-35.

Pain, M.T.G., Challis, J.H., 2001. The role of the heel pad and shank soft tissue during impacts: a further resolution of a paradox. Journal of Biomechanics 34, 327-333.

Perttunen, J., 2000. Biomechanical loading in the triple jump. Journal of Sports Sciences 18, 363370.

Riener, R., Edrich, T., 1999. Identification of passive elastic joint moments in the lower extremities. Journal of Biomechanics 32, 539-544.

Wilson, C., King, M.A., Yeadon, M.R., 2006. Determination of subject-specific model parameters for visco-elastic elements. Journal of Biomechanics 39, 1883-1890.

Wood, G.A., Jennings, L.S., 1979. On the use of spline functions in data smoothing. Journal of Biomechanics 12, 477-479.

Yeadon, M.R., 1990. The simulation of aerial movement-II. A mathematical inertia model of the human body. Journal of Biomechanics 23, 67-74.

Yeadon, M.R., Hiley, M.J., 2000. The mechanics of the backward giant circle on the high bar. Human Movement Science 19, 153-173.

Yeadon, M.R., King, M.A., Forrester, S.E., Caldwell, G.E., Pain, M.T.G., 2010. The need for muscle co-contraction prior to a landing. Journal of Biomechanics 43, 364-369. 
Yeadon. M.R., King, M.A., Wilson, C., 2006. Modelling the maximum voluntary joint torque / angular velocity relationship in human movement. Journal of Biomechanics 39, 476-482. 